\title{
Optimizing Hemodynamic Care in Neonatal Encephalopathy
}

Raymand Pang ${ }^{1}$, Alison Mintoft ${ }^{1}$, Rose Crowley ${ }^{2}$, Mark Sellwood ${ }^{2}$, Subhabrata Mitra $^{1}$, Nicola J Robertson ${ }^{1}$

1. Institute for Women's Health, University College London, London, UK

2. Department of Neonatology, University College London Hospital, London, UK

Corresponding Author:

Nicola J Robertson

Institute for Women's Health

University College London

London

WC1E 6HX

Tel: (+44) 2076796052

Email: n.robertson@ucl.ac.uk

\section{WORD COUNT: 5149 words + 4 Figures (200-250 words equivalent)}

\section{Abbreviations}

BP: blood pressure; CBF: Cerebral blood flow; CO: Cardiac output; ECMO:

Extracorporeal membrane oxygenation; FTOE: fractional tissue oxygen extraction; $\mathrm{HbD}$ : Hemoglobin difference; $\mathrm{HI}$ : Hypoxia-ischemia; $\mathrm{HbT}$ : Total hemoglobin; $\mathrm{HT}$ : Therapeutic Hypothermia; Lac/NAA: Lactate/N-acetyl aspartate; LV: Left ventricle; MABP: Mean arterial blood pressure; MRI: Magnetic resonance imaging; NE: Neonatal Encephalopathy; NIRS: Near infrared spectroscopy; PPI: Pressure passivity index; PPHN: Persistent pulmonary hypertension of the newborn; PVR: Pulmonary vascular resistance; $\mathrm{rSO}_{2}$ : Brain regional oxygen saturations; $\mathrm{RV}$ : Right ventricle; SVR: Systemic vascular resistance 


\section{Abstract}

Hemodynamic impairment occurs in up to $80 \%$ of infants with neonatal encephalopathy (NE). Not all infants benefit from therapeutic hypothermia (HT); there are some indications that the trajectory of brain injury might be modified by neurologic monitoring and early management over the first 72-hour period. It is also possible that optimizing hemodynamic management may further improve outomes. The coupling between cerebral blood flow and cerebral metabolism is disrupted in NE, increasing the vulnerability of the newborn brain to secondary injury. Hemodynamic monitoring is usually limited to blood pressure and functional echocardiographic measurements, which may not accurately reflect brain perfusion. This review explores the evidence base for hemodynamic assessment and management of infants with NE while undergoing HT. We discuss the literature behind a systematic approach to a baby with NE with the aim to define best therapies to optimize brain perfusion and reduce secondary injury.

\section{Keywords:}

Neonatal Encephalopathy, Cerebral Autoregulation, Cerebral Blood Flow, cardiovascular supportive therapies, therapeutic hypothermia, near infrared spectroscopy (NIRS) 


\subsection{Introduction}

Neonatal encephalopathy (NE) is an important cause of death and disability, affecting $1-3 / 1000$ births in the UK [1] and 10-25/1000 births in Sub-Saharan Africa [2]. The concept of neurocritical care has recently been taken up in many neonatal units around the world; such refinement of neurologic care is starting to show beneficial results. A recent implementation of a neurocritical care program for babies with NE was associated with decreased brain injury on magnetic resonance imaging (MRI), shortened hospital stay, and improved care of babies with moderate to severe NE [3]. One of the most critical periods on the hypoxic-ischemic encephalopathy care pathway (Fig 1) is the 72-hour period of therapeutic hypothermia (HT); it is plausible that the trajectory of brain injury might be modified by optimizing hemodynamic as well as neurologic monitoring and management. Hemodynamic instability has been described to occur in up to $80 \%$ of infants enrolled in clinical cooling trials with similar rates between those cooled and normothermic [4].

In this review we focus on hemodynamic compromise and instability in babies with moderate to severe NE secondary to intrapartum hypoxia ischemia $(\mathrm{HI})$, discussing evidence-based assessment and management. The cardiovascular effects of intrapartum $\mathrm{HI}$ are significant, with reduced myocardial perfusion and decreased myocardial contractility occurring as part of the primary insult, followed by challenges to hemodynamic stability with redistribution of blood to the injured brain. One-third of asphyxiated neonates develop transient myocardial ischemia, sometimes leading to myocardial infarction [5]. Although HT has transformed the outcomes of babies with moderate to severe NE, not all babies benefit. With the current practice of $\mathrm{HT}$, mortality of NE has been reduced from $25 \%$ to $9 \%$ and disability from $20 \%$ to around $16 \%$ with 
a reduction in the rate of cerebral palsy [6]. Intellectual impairment may remain even in the absence of cerebral palsy [7] and ways to improve outcomes are urgently needed. The relation between altered hemodynamic profiles in the subacute phase of NE and optimizing brain protection is unclear. It is common for inotropes to be started when a baby surviving a major resuscitation at birth develops low blood pressure (BP) or signs of low cardiac output (CO). We explore a systematic approach to a baby with NE by assessing the cause of the hemodynamic disturbances to provide individualized therapy to optimize brain perfusion and reduce secondary injury. As routine therapy for moderate to severe NE involves $72 \mathrm{~h}$ of $\mathrm{HT}$, we discuss the hemodynamic effects of cooling and rewarming and how to optimize cardiovascular support and brain perfusion.

\subsection{Pathophysiology of Cardiovascular Dysfunction in NE}

The impact of $\mathrm{HI}$ on the cardiovascular system was comprehensively reviewed by Popescu et al 2020 [8]. In brief, prolonged HI leads to myocardial ischemia despite initial compensatory mechanisms to ensure preferential blood flow to the heart and brain. Although neonatal cardiomyocytes are relatively adapted to a hypoxic environment through glycolytic metabolism, antioxidant defense mechanisms are underdeveloped and place the heart at unique vulnerability to reperfusion injury. After resuscitation, re-oxygenation and reperfusion lead to the generation of reactive oxygen species activating apoptotic pathways.

The transition from fetal to neonatal circulation is characterized by a fall in pulmonary vascular resistance, left-to-right flow reversal across the ductus arteriosus (DA) and foramen ovale, and eventual closure of the fetal shunts. Hypoxia leads to a disequilibrium in favor of endothelin 1 production, resulting in inhibition of nitric oxide 
synthase, reduction in nitric oxide (NO) production, and vasoconstriction, subsequently resulting in persistence of high pulmonary vascular resistance (PVR) and in some cases, persistent pulmonary hypertension of the newborn (PPHN). In a secondary analysis of two cooling studies [9], PPHN affected $20-22 \%$ of infants with NE and was associated with a significant increase in mortality. Factors associated with PPHN in NE include concurrent lung pathology (meconium aspiration, pulmonary hemorrhage), severe postnatal acidosis, and sepsis. PPHN develops as a result of a vicious cycle of raised PVR, reduced pulmonary blood flow, reduced oxygenation and right-to-left shunting across the DA secondary to pulmonary hypertension. Both left ventricular (LV) and right ventricular (RV) dysfunction can occur [10]. Reduction in pulmonary perfusion leads to reduction in pulmonary venous return to the left atrium, reducing $L V$ filling and output. The increase in RV afterload can lead to RV dilation and failure, further compromising pulmonary blood flow, thereby reducing systemic and cerebral oxygen delivery.

The capacity of the neonatal myocardium to respond to the hemodynamic changes during transitional postnatal adaptation and following $\mathrm{HI}$ is limited. The cellular organization of the newborn myocardium, consisting of disorganized myofibrils with fewer contractile components and excitation-contraction communication between cardiomyocytes, is underdeveloped. Furthermore, the transition from inefficient anaerobic metabolism in fetal life to more effective aerobic metabolism is delayed after hypoxia, reducing the contractile capacity of heart. The underdeveloped autonomic nervous system at term and adrenal insufficiency in the context of the unwell infant may further contribute to the reduced capacity to respond to hemodynamic compromise. 


\subsection{Assessment of cardiovascular status and cerebral perfusion in NE}

Cerebral perfusion is dependent upon the blood pressure gradient (perfusion pressure) and cerebral vascular tone. Cerebral blood flow (CBF), function, and metabolism are tightly coupled and the uncoupling that occurs in $\mathrm{HI}$ is implicated in brain injury [11]. This is compounded by the fall in CBF on the first day of life before a progressive rise over several days, depending on the degree of injury [12], increasing the vulnerability of the newborn brain to ischemia followed by hyperperfusion. Therefore, the ability to monitor and optimize cerebral perfusion is critical for the prevention of ischemic and reperfusion injury.

In practice, assessing hemodynamic function in infants is often limited to measurement of blood pressure (BP), heart rate, and clinical indicators such as capillary refill time, core/peripheral temperature difference, and urine output. BP is often the main driver influencing medical interventions. However, the critical threshold for BP to maintain adequate cerebral perfusion is unknown and may change with different disease states [13]. Blood pressure is a dependent variable relying upon the product of two independent variables regulated by the body: cardiac output (CO) and systemic vascular resistance (SVR). $\mathrm{CO}$ is determined by the product of stroke volume (SV) and heart rate and can be measured by targeted neonatal echocardiography, a bedside tool used to assess cardiovascular performance and refine therapeutic interventions in this high risk group [14, 15]. SVR can be derived from knowledge of the mean arterial $\mathrm{BP}$ (MABP) and CO; however, systemic blood flow may not always directly relate to organ-specific blood flow.

Cerebral autoregulation maintains a steady $\mathrm{CBF}$ in response to changes in cerebral perfusion pressure, protecting the brain from injury. Cerebral autoregulation is influenced by multiple pathologic factors, increasing the susceptibility of the 
developing brain to injury secondary to changes in systemic blood pressure [13]. Cerebral vascular tone in the healthy brain is maintained by a balance between vasodilation and vasoconstriction. CBF is kept within a tight window, with an upper and lower limit of autoregulation (Fig 2), often measured as pressure reactivity. Pressure passivity results in blood pressure dependent changes in CBF and occurs when BP falls outside the autoregulatory range. A drop in BP below the critical closing pressure renders the developing brain susceptible to ischemic hypoperfusion injury, whereas hypertension can lead to hyperperfusion injury.

The threshold for cerebral autoregulation varies with gestation, postnatal age, and vasoactive factors [16]. In the healthy neonate, the autoregulatory response in CBF is tightly coupled to the metabolic demand. Although BP plays an important role in CBF, Greisen et al (2005) reported that the relationship between $\mathrm{pCO}_{2}$ and $\mathrm{CBF}$ is exponential and might be more important than pressure-flow reactivity [17]. Vasodilation also occurs in response to low $\mathrm{PaO}_{2}$, high $\mathrm{pCO}_{2}$, low glucose, low $\mathrm{Ca}^{2+}$, elevated $\mathrm{K}^{+}$, elevated $\mathrm{H}^{+}$, acidemia secondary to hypercarbia or lactic acidosis, and elevated adenosine and prostanoids $[16,17]$.

Following severe perinatal $\mathrm{HI}$, initial hypoperfusion of the brain is followed by hyperperfusion in a predictable pattern. This increased CBF (also termed luxury perfusion or hyperperfusion) on day 2-3 in NE infants reflects a state of maximal vasodilation in response to the release of vasoactive factors and is associated with adverse neurodevelopmental outcome [18] even with HT [19]. It is unclear if this period of hyperperfusion is a manifestation of the severity of the underlying insult or whether the reperfusion contributes to the severe injury. There is no evidence that aggressive cardiovascular management of hypotension reduces brain injury during the evolution of injury in NE. 
Near-infrared spectroscopy (NIRS) offers the ability to continuously assess cerebral regional blood flow, regional oxygenation, and mitochondrial oxidative metabolism at the bedside. It can play an important role when combined with functional echocardiography in guiding therapeutic interventions in NE to prevent secondary brain injury. In a recent phase II, single masked randomized clinical trial of preterm infants, a NIRS based management approach reduced the median burden of hypoxia and hyperoxia by $58 \%(95 \% \mathrm{Cl} 35-73 \%, \mathrm{p}<0.001)$ compared to clinical care without NIRS [20]. Although similar studies in term infants are yet to be published, impaired cerebral autoregulation in NE has been demonstrated using NIRS [11]. Massaro et al [21] used the pressure passivity index (PPI) derived from the coherence between hemoglobin difference $(\mathrm{HbD}$, a measurement of intravascular oxygenation and a surrogate marker of $\mathrm{CBF}$ ) and MABP and showed infants who died or developed moderate to severe injury on MRI exhibited longer and more significant periods of cerebral pressure passivity. Impairment of pressure reactivity can result in uncoupling of CBF and cerebral energy metabolism leading to further brain injury. Using continuous measurement of NIRS based reactivity indices, the changes in cerebrovascular autoregulation can be monitored and it is possible to identify the optimal blood pressure (MABP ${ }_{\mathrm{OPT}}$ ) at the point of most preserved autoregulatory capacity. Howlett et al, Burton et al, and Lee et al showed that a MABP $<$ MABPOPT was associated with an increased severity of brain injury on MRI and poor neurodevelopmental outcomes [22-24]. However, MABPOPT in these studies ranged from 45 to $55 \mathrm{mmHg}$. The advantage of the use of a cerebral autoregulatory index is individualized care in real-time. 
Continuous monitoring using cerebral oximetry with NIRS in NE can also predict neurological outcomes [25]. Post-HI drop in cerebral oxygenation is less pronounced in infants with subsequent brain injury compared to infants without brain injury [26], likely reflecting luxury perfusion and impaired oxygen utilization resulting from mitochondrial dysfunction. Studies in both pre-cooling and cooling era indicated a significant difference in cerebral oxygenation following NE between groups with or without evidence of brain injury [26, 27]. The sensitivity and specificity of cerebral oxygenation to predict the adverse outcome within the first 10 hours of HT were 100 and $83 \%$ in the study by Peng et al. [26]. Lemmers et al identified the highest predictive value at $24-30$ hours of life with a sensitivity of $92 \%$ and specificity of $64 \%$ [27].

Mitra et al used wavelet-based metabolic (cytochrome c oxidase-MABP semblance) and hemodynamic ( $\mathrm{HbD}-\mathrm{MABP}$ semblance) reactivity indices in a cohort of infants undergoing HT. Disturbed cerebral metabolic reactivity was associated with higher evidence of injury on MRI, thalamic Lac/NAA on proton magnetic resonance spectroscopy (MRS) and poor neurodevelopmental outcome [28]. Recently, Giesinger and colleagues used a combination of functional echocardiography, NIRS, and MRI to assess the relationship between myocardial dysfunction and the severity of brain injury on MRI or death [14]. Right ventricular impairment independently predicted death and/or abnormal MRI. In infants with impaired RV function on echocardiography, brain regional oxygen saturations $\left(\mathrm{rSO}_{2}\right)$ on $\mathrm{NIRS}$ were higher and fractional tissue oxygen extraction (FTOE) lower, reflecting reduced metabolic activity in the brain from widespread mitochondrial dysfunction. A NIRS-based clinical approach holds promise in the management of $\mathrm{NE}$, as this may assist to improve stability in cerebral hemodynamics and metabolism following $\mathrm{HI}$ injury. 
Although continuous monitoring of cerebral oxidative metabolism using cytochrome-c oxidase or cerebral metabolic rate of oxygen $\left(\mathrm{CMRO}_{2}\right)$ from the newer optical devices are yet to come into clinical practice, important information can be obtained from commercially available cerebral oximetry to monitor the changes in brain pathophysiology in NE. Heterogeneity in sensors and methodology used in different NIRS devices as well as variation in terminology used by manufacturers can complicate the interpretation of findings between studies, but most of the commercially available oximetry monitors have shown a reasonable correlation between the measured cerebral oxygenation values [11].

\subsection{Cardiac compromise and neurologic outcomes}

Data on the long-term neurodevelopmental outcomes among infants with cardiovascular compromise in NE are limited and variable. Shah et al [29] reported no association between cardiovascular injury and adverse neurodevelopmental outcomes after intrapartum asphyxia. The definition of cardiac compromise was nonspecific in this study, as it included ischemic changes on ECG which are also observed in well newborns [30].

No clinical trials to date have investigated the therapeutic benefit of inotropic support on hemodynamic instability in relation to outcomes in infants with NE. DiSessa and colleagues [31] reported a small randomized controlled trial investigating the cardiovascular effects of dopamine vs placebo in 14 infants following HI. No treatment benefit was observed, although infants required $M A B P>50 \mathrm{mmHg}$ to be eligible for entry into the trial and thus were not hemodynamically compromised. Two recent studies in cooled infants with NE reported abnormal neuro-imaging findings in hypotensive infants receiving inotropes. These studies used MABP $<40 \mathrm{mmHg}$ as the 
main determinant of medical intervention. Mohammad and colleagues [32] observed that receipt of inotropic support in the first $72 \mathrm{~h}$ of life was associated with a three-fold increase in the odds of neonatal mortality or an abnormal MRI. The group receiving inotropes were also more likely to have severe disease; however, hypotension remained a significant predictor of adverse MRI findings after adjustment. This study also showed an increase in mortality and brain injury on EEG and MRI among infants with resistant hypotension, receiving more than one inotrope. Al Balushi and colleagues [33] similarly reported hypotension treated with inotropes as an independent predictor of brain injury on MRI in infants with moderate to severe NE. Whether these findings relate to the severity of the primary $\mathrm{HI}$ injury or the consequences of treatment remains unknown. Bashir et al reported an increased risk of intraparenchymal hemorrhage (OR 3.3, 95\% Cl 1.2-9.2) among infants requiring inotropic support even after adjusting for NE severity [34] but were unable to reach a conclusion on whether inotropes were primarily responsible for the hemorrhage. The evidence base in the choice of inotropes to manage hypotensive infants with NE is limited. Al Balushi et al [33] reported an increase in brain injury on MRI among infants treated with dobutamine while Mohammed et al [32] and Bashir et al [34] reported increased brain injury among infants treated with dopamine and dobutamine. Although these studies were not designed or powered to assess the inotrope of choice, they highlight the lack of evidence about the best management of hypotensive infants with NE.

Overall, the data suggest that treating hypotension as opposed to poor $\mathrm{CO}$ or decreased flow has not improved mortality and morbidity in NE. Management of hemodynamic instability is a balance between preventing hypoperfusion injury as a result of systemic hypotension and prevention of hyperperfusion injury as a result of 
luxury cerebral perfusion. Targeted neonatal echocardiography and regional cerebral perfusion with NIRS may be the appropriate approach for improving outcome in critically ill neonates with NE.

\subsection{Cardiovascular impact of therapeutic hypothermia}

For a critical period of $72 \mathrm{~h}$ in the sub-acute phase of NE, babies with moderate to severe NE undergo $\mathrm{HT}$ to $33.5^{\circ} \mathrm{C}$. The mechanisms of brain protection from $\mathrm{HT}$ are multi-factorial [35]; animal studies also suggest a cardioprotective effect of HT [36]. Typical clinical signs of cardiovascular compromise during HT may not be reliably elicited as a result of the physiologic response to hypothermia. Vasoconstriction occurs, reducing the capillary refill time. Core-peripheral temperature difference is disturbed by active whole-body cooling. Urine output may be reduced in response to $\mathrm{HI}$ injury. Sinus bradycardia occurs because of less influence of the sympathetic nervous system on heart rate and slowed diastolic repolarization of the sino-atrial node (Fig 3). This relative bradycardia may result in less myocardial muscle energy requirement leading to protection, whereas the use of chronotropic cardiovascular therapies may increase metabolic requirements. The reduction in HR with HT leads to a $60-70 \%$ decrease in left ventricular output (LVO); as the body's metabolic activity is reduced with $\mathrm{HT}$, this lower $\mathrm{CO}$ is likely to meet with the metabolic demands of the NE infant and may not suggest decompensated shock. HT is not associated with an increased risk of hypotension from the $\mathrm{HT}$-induced vasoconstriction. Short term studies of brain MRI have shown that impaired LVO during cooling was not associated with adverse MRI outcomes [14, 37]. 
In animal studies [38], hypothermia was associated with increase in PVR; however, heightened risk of PPHN was not observed in RCTs for HT [39]. It is important to note that these studies were not powered to detect severity of PPHN, and in some trials, PPHN formed part of the exclusion criteria. With the risk of lower preductal CO (as a result of impaired $\mathrm{RV}$ function and reduced $\mathrm{LA}$ preload) and reduced $\mathrm{CBF}$, several studies show PPHN in NE is associated with an abnormal findings on regional brain oxygenation [40] and MRI $[41,42]$. In a case series of infants with NE, the most severe impairment in cerebral oxygenation was associated with clinical signs of right-to-left shunting [40]. However, interventions including inotropes to augment $\mathrm{CO}$ and inhaled nitric oxide, which may increase venous return could also exacerbate reperfusion injury. A normal MRI scan is associated with lower CBF (which is even lower during $\mathrm{HT}$ ), whereas an abnormal MRI scan is associated with a higher CBF (which typically progressively rises during HT) [43, 44] (Fig 3). Hyperperfusion and associated hyperoxemia are likely secondary factors which exacerbate injury and manipulating CBF into the optimal range may optimize outcomes.

During rewarming, Noori and colleagues showed that CO significantly increases secondary to an increase in HR rather than stroke volume; this is associated with an increase in renal blood flow [45]. The decrease in SVR and diastolic BP, along with the increase in $\mathrm{CO}$ and systolic $\mathrm{BP}$, results in an overall reduction in MABP of $8 \mathrm{mmHg}$ (Fig 3). Importantly the pattern of redistribution of CBF to the brain seen during HT persists. It is important to note that inotropes infused during the rewarming period may be mobilized from sequestered tissues and have an exaggerated effect during rewarming. Periods of desaturation have been reported to be more frequent during the rewarming period and infants may be at increased risk of pulmonary hypertensive crisis $[40,46]$ requiring ECMO. Rewarming is therefore a vulnerable period, and it is 
important to avoid large fluctuations in CBF during this time. Indeed, pre-emptive adjustment of inotropes may be necessary to avoid iatrogenic hypertension and increased CBF.

\subsection{Management Approach}

The approach to cardiovascular care needs to take into account the phase of HT or rewarming. PPHN and both RV and LV dysfunction may co-exist in NE and the use of functional echocardiography for cardiovascular assessment can help guide management. Similarly, the use of cerebral NIRS can guide the clinician regarding the continuous changes in cerebral hemodynamics and oxidative metabolism following injury [11], and presents an early opportunity to assess the injury severity at the bedside. Cardiac and cerebrovascular management using functional echocardiography and cerebral NIRS together allows assessment in the effect of $\mathrm{HI}$ injury as well as the response and progress during HT.

The current evidence behind the use of inotropes in NE babies has been reviewed by the McNamara group [15], Joynt and Cheung [47], Dempsey and Rabe [48] and Gupta and Singh [49].

The key principles in relation to approaching cardiovascular care in NE are as follows:

- Avoid aggressive volume resuscitation except when there is evidence of hypovolemia given the association between increased CBF and poor outcome

- Balance the risks of using inotropes with the potential iatrogenic exacerbation of reperfusion injury.

- Stabilization of an infant with NE with oxygenation problems should precede $\mathrm{HT}$ exposure as HT may increase the degree of pulmonary vasoconstriction. 
- The specific target of the cardiovascular medication needs to be assessed ( $\mathrm{CO}$, $\mathrm{BP}, \mathrm{PVR}$ ) with the aim to improve oxygenation, organ perfusion, and cardiac function. The therapy needs to be balanced against potential adverse effects.

- In the absence in availability of echocardiography and NIRS, the optimal BP based on clinical studies [22-24] is likely within normal range, however BP does not always accurately reflect CBF therefore caution needs to be taken when using a BP targeted approach.

Targeted neonatal echocardiography can be utilized to help guide intervention as follows [49]:

- In the presence of myocardial dysfunction and increase SVR, dobutamine may be the most appropriate first line agent of choice to increase stroke volume and $\mathrm{CO}$ and reduce afterload.

- In the presence of myocardial dysfunction and reduced SVR, use agents with both inotropic and vasopressor effect

- In severe NE, adrenal insufficiency may present as refractory hypotension and the addition of hydrocortisone may improve cardiovascular function.

- In the presence of PPHN, serial echocardiographic assessment of the hemodynamics is essential. Dobutamine is also an appropriate choice as it improves $\mathrm{CO}$ and may provide some support in pulmonary blood flow. Infants with RV impairment and restrictive DA may benefit from low-dose prostaglandin $E_{1}$ infusion to maintain patency and offload the RV. In addition to inotropic support, inhaled nitric oxide may be required to reduce PVR. However, caution must be taken to prevent hyperperfusion injury.

\subsection{Conclusion}


The transitional circulatory physiology of the newborn places term infants with moderate to severe NE at increased risk of decompensated shock and secondary brain injury. However, current hemodynamic monitoring is routinely limited to $\mathrm{BP}$, which is a poor measure of CBF. No study has shown benefit from the use of inotropes in cardiovascular compromise in NE. Loss of cerebral autoregulation places the newborn brain at increased risk of injury and is seen in surviving babies with adverse outcome after NE. Hyperperfusion and associated hyperoxia are factors which are likely to exacerbate secondary injury. Rewarming is a vulnerable period when BP rises for physiologic reasons, but as inotropes become less sequestered in tissues, it is important to avoid iatrogenic hypertension and excessive CBF. Regional NIRS offers the potential to monitor cerebral perfusion and its coupling to cerebral metabolism. A combination of real time multimodal bedside assessment including cerebral NIRS and targeted neonatal functional echocardiography, may help to individualize therapy and improve outcomes.

\section{Practice Points}

- Perinatal hypoxia ischemia leading to neonatal encephalopathy can disrupt normal cerebral autoregulation, increasing the vulnerability of the newborn brain to secondary brain injury from hypo- and hyperperfusion

- Using MABP $<40 \mathrm{mmHg}$ as the threshold for requiring inotropes in a baby with NE is problematic as this does not directly relate to brain perfusion and oxygenation.

- One of the most critical periods on the NE roadmap, is the $72 \mathrm{~h}$ period of HT when the trajectory of brain injury might be modified by optimizing hemodynamic and well as neurological monitoring and management. 
- Hyperperfusion and associated hyperoxia are factors which are likely to exacerbate secondary injury; it is plausible that manipulating CBF into the optimal range may optimize outcomes.

- Rewarming is a vulnerable period when MABP rises for physiological reasons, but as inotropes become less sequestered in tissues, it is important to avoid iatrogenic hypertension and excessive CBF.

\section{Research Directions}

- Identify measures of adequate cerebral blood flow on a case by case basis to individualize care and prevent secondary brain injury in the hemodynamically compromised infant.

- Comparison and validation of functional echocardiography and continuous near infrared spectroscopy (NIRS) monitoring; a knowledge of the relevance of and relationship between these techniques may assist in individualized therapy to prevent hypo- and hyperperfusion injury in NE

- Careful assessment of the most appropriate inotropic support in hemodynamically compromised infants to support cerebral perfusion 


\section{Figure Legends}

FIGURE 1: Hypoxic Ischemic Encephalopathy road map highlighting the critical 72hour window

FIGURE 2: Cerebral Autoregulation

FIGURE 3: Cardiovascular impact of therapeutic hypothermia and rewarming and associations with MRI outcomes

FIGURE 4: Pragmatic Approach to Cardiovascular Impairment for infants with NE. Adapted from Giesinger et al 2017 [15] and Joynt and Cheung 2018 [47] 


\section{References}

[1] Gale C, Statnikov Y, Jawad S, Uthaya S, Modi N, group Blew. Neonatal brain injuries in England: population-based incidence derived from routinely recorded clinical data held in the National Neonatal Research Database. Arch Dis Child Fetal Neonatal Ed. 2018;103:F3016.

[2] Lee A, Kozuki N, Blencowe H, Vos T, Bahalim A, Darmstadt G, et al. Intrapartum-related neonatal encephalopathy incidence and impairment at regional and global levels for 2010 with trends from 1990. Pediatr Res. 2013;74:50-72.

[3] Roychoudhury S, Esser MJ, Buchhalter J, Bello-Espinosa L, Zein H, Howlett A, et al. Implementation of Neonatal Neurocritical Care Program Improved Short-Term Outcomes in Neonates With Moderate-to-Severe Hypoxic Ischemic Encephalopathy. Pediatr Neurol. United States: (C) 2019 Elsevier Inc; 2019. p. 64-70.

[4] Azzopardi D, Strohm B, Edwards A, Dyet L, Halliday H, Juszczak E, et al. Moderate hypothermia to treat perinatal asphyxial encephalopathy. N Engl J Med. 2009;361:1349-58. [5] Armstrong K, Franklin O, Sweetman D, Molloy E. Cardiovascular dysfunction in infants with neonatal encephalopathy. Arch Dis Child. 2012;97:372-5.

[6] Jary S, Lee-Kelland R, Tonks J, Cowan F, Thoresen M, Chakkarapani E. Motor performance and cognitive correlates in children cooled for neonatal encephalopathy without cerebral palsy at school age. Acta Paediatr. 2019;108:1773-80.

[7] Lee-Kelland R, Jary S, Tonks J, Cowan F, Thoresen M, Chakkarapani E. School-age outcomes of children without cerebral palsy cooled for neonatal hypoxic-ischaemic encephalopathy in 2008-2010. Arch Dis Child Fetal Neonatal Ed. 2020;105:8-13.

[8] Popescu MR, Panaitescu AM, Pavel B, Zagrean L, Peltecu G, Zagrean AM. Getting an Early Start in Understanding Perinatal Asphyxia Impact on the Cardiovascular System. Front Pediatr. 2020;8:68.

[9] Lakshminrusimha S, Shankaran S, Laptook A, McDonald S, Keszler M, Van Meurs K, et al. Pulmonary Hypertension Associated with Hypoxic-Ischemic Encephalopathy-Antecedent Characteristics and Comorbidities. J Pediatr. 2018;196:45-51 e3.

[10] Aggarwal S, Natarajan G. Biventricular function on early echocardiograms in neonatal hypoxic-ischaemic encephalopathy. Acta Paediatr. 2017;106:1085-90.

[11] Mitra S, Bale G, Meek J, Tachtsidis I, Robertson NJ. Cerebral Near Infrared Spectroscopy Monitoring in Term Infants With Hypoxic Ischemic Encephalopathy-A Systematic Review. Front Neurol. 2020;11:393.

[12] Noori S, Wlodaver A, Gottipati V, McCoy M, Schultz D, Escobedo M. Transitional changes in cardiac and cerebral hemodynamics in term neonates at birth. J Pediatr. 2012;160:943-8.

[13] Rhee CJ, da Costa CS, Austin T, Brady KM, Czosnyka M, Lee JK. Neonatal cerebrovascular autoregulation. Pediatr Res. 2018;84:602-10.

[14] Giesinger RE, El Shahed Al, Castaldo MP, Breatnach CR, Chau V, Whyte HE, et al. Impaired Right Ventricular Performance Is Associated with Adverse Outcome after Hypoxic Ischemic Encephalopathy. Am J Respir Crit Care Med. 2019;200:1294-305.

[15] Giesinger RE, Bailey L, Deshpande P, McNamara PJ. Hypoxic-Ischemic Encephalopathy and Therapeutic Hypothermia: The Hemodynamic Perspective. J Pediatr. United States2017. p. 22-30 e2.

[16] Volpe JJ, Inder TE, Darras BT, de Vries LS, du Plessis AJ, Neil JJ, et al. Volpe's Neurology of the Newborn2018. 
[17] Greisen G. Autoregulation of cerebral blood flow in newborn babies. Early Hum Dev. 2005;81:423-8.

[18] Wintermark P, Moessinger AC, Gudinchet F, Meuli R. Perfusion-weighted magnetic resonance imaging patterns of hypoxic-ischemic encephalopathy in term neonates. J Magn Reson Imaging. 2008;28:1019-25.

[19] Wintermark P, Hansen A, Gregas MC, Soul J, Labrecque M, Robertson RL, et al. Brain perfusion in asphyxiated newborns treated with therapeutic hypothermia. AJNR Am J Neuroradiol. 2011;32:2023-9.

[20] Hyttel-Sorensen S, Pellicer A, Alderliesten T, Austin T, van Bel F, Benders M, et al. Cerebral near infrared spectroscopy oximetry in extremely preterm infants: phase II randomised clinical trial. BMJ. 2015;350:g7635.

[21] Massaro AN, Govindan RB, Vezina G, Chang T, Andescavage NN, Wang Y, et al. Impaired cerebral autoregulation and brain injury in newborns with hypoxic-ischemic encephalopathy treated with hypothermia. J Neurophysiol. 2015;114:818-24.

[22] Howlett JA, Northington FJ, Gilmore MM, Tekes A, Huisman TA, Parkinson C, et al. Cerebrovascular autoregulation and neurologic injury in neonatal hypoxic-ischemic encephalopathy. Pediatr Res. 2013;74:525-35.

[23] Burton VJ, Gerner G, Cristofalo E, Chung SE, Jennings JM, Parkinson C, et al. A pilot cohort study of cerebral autoregulation and 2-year neurodevelopmental outcomes in neonates with hypoxic-ischemic encephalopathy who received therapeutic hypothermia. BMC Neurol. 2015;15:209.

[24] Lee JK, Poretti A, Perin J, Huisman T, Parkinson C, Chavez-Valdez R, et al. Optimizing Cerebral Autoregulation May Decrease Neonatal Regional Hypoxic-Ischemic Brain Injury. Dev Neurosci. 2017;39:248-56.

[25] Abelian A, Mund T, Curran MD, Savill SA, Mitra N, Charan C, et al. Towards accurate exclusion of neonatal bacterial meningitis: a feasibility study of a novel 16S rDNA PCR assay. BMC Infect Dis. 2020;20:441.

[26] Peng S, Boudes E, Tan X, Saint-Martin C, Shevell M, Wintermark P. Does near-infrared spectroscopy identify asphyxiated newborns at risk of developing brain injury during hypothermia treatment? Am J Perinatol. 2015;32:555-64.

[27] Lemmers PM, Zwanenburg RJ, Benders MJ, de Vries LS, Groenendaal F, van Bel F, et al. Cerebral oxygenation and brain activity after perinatal asphyxia: does hypothermia change their prognostic value? Pediatr Res. 2013;74:180-5.

[28] Mitra S, Kendall GS, Bainbridge A, Sokolska M, Dinan M, Uria-Avellanal C, et al. Proton magnetic resonance spectroscopy lactate/ $\mathrm{N}$-acetylaspartate within 2 weeks of birth accurately predicts 2-year motor, cognitive and language outcomes in neonatal encephalopathy after therapeutic hypothermia. Arch Dis Child Fetal Neonatal Ed. 2019;104:F424-F32.

[29] Shah P, Riphagen S, Beyene J, Perlman M. Multiorgan dysfunction in infants with postasphyxial hypoxic-ischaemic encephalopathy. Arch Dis Child Fetal Neonatal Ed.

2004;89:F152-5.

[30] Jedeikin R, Primhak A, Shennan AT, Swyer PR, Rowe RD. Serial electrocardiographic changes in healthy and stressed neonates. Arch Dis Child. 1983;58:605-11.

[31] DiSessa TG, Leitner M, Ti CC, Gluck L, Coen R, Friedman WF. The cardiovascular effects of dopamine in the severely asphyxiated neonate. J Pediatr. 1981;99:772-6. 
[32] Mohammad K. Hemodynamic instability associated with increased risk of death or brain injury in neonates with hypoxic ischemic encephalopathy. Journal of Neonatal-Perinatal Medicine. 2016;9:357-62.

[33] Al Balushi A, Barbosa Vargas S, Maluorni J, Sanon PN, Rampakakis E, Saint-Martin C, et al. Hypotension and Brain Injury in Asphyxiated Newborns Treated with Hypothermia. Am J Perinatol. 2018;35:31-8.

[34] Bashir RA, Vayalthrikkovil S, Espinoza L, Irvine L, Scott J, Mohammad K. Prevalence and Characteristics of Intracranial Hemorrhages in Neonates with Hypoxic Ischemic Encephalopathy. Am J Perinatol. 2018;35:676-81.

[35] Liu L, Yenari M. Therapeutic hypothermia: neuroprotective mechanisms. Front Biosci. 2007;12.

[36] Liu X, Tooley J, Løberg EM, Suleiman MS, Thoresen M. Immediate hypothermia reduces cardiac troponin I after hypoxic-ischemic encephalopathy in newborn pigs. Pediatr Res. 2011;70:352-6.

[37] More K, Sakhuja P, Giesinger R, Ting J, Keyzers M, Sheth J, et al. Cardiovascular Associations with Abnormal Brain Magnetic Resonance Imaging in Neonates with Hypoxic Ischemic Encephalopathy Undergoing Therapeutic Hypothermia and Rewarming. American Journal of Perinatology. 2018.

[38] Benumof JL, Wahrenbrock EA. Dependency of hypoxic pulmonary vasoconstriction on temperature. J Appl Physiol Respir Environ Exerc Physiol. 1977;42:56-8.

[39] Jacobs SE, Berg M, Hunt R, Tarnow-Mordi WO, Inder TE, Davis PG. Cooling for newborns with hypoxic ischaemic encephalopathy. Cochrane Database Syst Rev. 2013:CD003311. [40] Gagnon MH, Wintermark P. Effect of persistent pulmonary hypertension on brain oxygenation in asphyxiated term newborns treated with hypothermia. J Matern Fetal Neonatal Med. 2016;29:2049-55.

[41] Jain A, McNamara PJ. Persistent pulmonary hypertension of the newborn: Advances in diagnosis and treatment. Semin Fetal Neonatal Med. Netherlands: Crown (c) 2015. Published by Elsevier Ltd; 2015. p. 262-71.

[42] More KS, Sakhuja P, Giesinger RE, Ting JY, Keyzers M, Sheth JN, et al. Cardiovascular Associations with Abnormal Brain Magnetic Resonance Imaging in Neonates with Hypoxic Ischemic Encephalopathy Undergoing Therapeutic Hypothermia and Rewarming. Am J Perinatol. 2018;35:979-89.

[43] Hochwald O, Jabr M, Osiovich H, Miller SP, McNamara PJ, Lavoie PM. Preferential cephalic redistribution of left ventricular cardiac output during therapeutic hypothermia for perinatal hypoxic-ischemic encephalopathy. J Pediatr. 2014;164:999-1004 e1.

[44] Kumagai T, Higuchi R, Higa A, Tsuno Y, Hiramatsu C, Sugimoto T, et al. Correlation between echocardiographic superior vena cava flow and short-term outcome in infants with asphyxia. 2013;89:307-10.

[45] Wu TW, Tamrazi B, Soleymani S, Seri I, Noori S. Hemodynamic Changes During Rewarming Phase of Whole-Body Hypothermia Therapy in Neonates with Hypoxic-Ischemic Encephalopathy. J Pediatr. United States: (C) 2018 Elsevier Inc; 2018. p. 68-74 e2.

[46] Shah SK, Khan AM, Cox CS, Jr. Pulmonary hypertensive crisis requiring ECMO associated with re-warming from whole body hypothermia for hypoxic ischemic encephalopathy:

clinical observations from a case series. Eur J Pediatr Surg. 2010;20:205-6.

[47] Joynt C, Cheung PY. Cardiovascular Supportive Therapies for Neonates With Asphyxia A Literature Review of Pre-clinical and Clinical Studies. Front Pediatr. 2018;6:363. 
[48] Dempsey E, Rabe H. The Use of Cardiotonic Drugs in Neonates. Clin Perinatol. United States: (C) 2019 Elsevier Inc; 2019. p. 273-90.

[49] Gupta S, Singh Y. Hemodynamics in the Asphyxiated Neonate and Effects of Therapeutic Hypothermia. 2018. p. 503-20. 